\title{
Breve caracterização dos tipos de negação em Mehinaku (Arawák)
}

DOI: http://dx.doi.org/10.21165/el.v48i3.2136

\author{
Angel Humberto Corbera Mori ${ }^{1}$ \\ Jackeline do Carmo Ferreira ${ }^{2}$
}

\section{Resumo}

A negação se manifesta nas línguas de duas maneiras: (i) como construção morfológica ou negação sintética e (ii) como construção sintática ou negação analítica. Tipologicamente, uma língua usa diversas estratégias para derivar uma oração negativa, tais como: (i) a ocorrência de verbos negativos; (ii) o uso de partículas negativas; (iii) o emprego de afixos; (iv) e a ocorrência de morfemas negativos com propriedades nominais. Neste artigo, apresenta-se uma breve análise das maneiras de se expressar negação na língua Mehinaku, uma língua da família Arawák. Além da negação padrão, inclui-se a descrição da construção proibitiva, da negação existencial e do uso do prefixo 'privativo' \{ma-\}, um sufixo derivacional, reflexo da proto-forma *ma-, e o 'atributivo' $\{k a-\}$. A análise se baseia em dados primários coletados em trabalhos de campo junto aos falantes da língua Mehinaku, das aldeias Utawana e Kaupüna, no Território Indígena do Xingu, Mato Grosso.

Palavras-chave: línguas Arawák; língua Mehinaku; negação padrão; tipologia da negação.

1 Universidade Estadual de Campinas (UNICAMP), Campinas, São Paulo, Brasil; corbera@uol.com.br; https://orcid.org/0000-0003-1712-6550

2 Universidade Estadual de Campinas (UNICAMP), Campinas, São Paulo, Brasil; jackelinedocarmoferreira@gmail.com; https://orcid.org/0000-0001-6332-7535 


\section{Brief characterization of the types of negation in Mehinaku (Arawak)}

\section{Abstract}

Negation manifests itself in languages in two ways: (i) as morphological construction or synthetic negation, and (ii) as syntactic construction or analytic negation. Typically, a language uses several strategies to derive a negative sentence, such as: (i) the occurrence of negative verbs; (ii) the use of negative particles; (iii) the use of affixes; (iv) and the occurrence of negative morphemes with nominal properties. In this paper, we present a brief analysis of the ways to express negation in the Mehinaku language, a language of the Arawak family. In addition to the standard negation, a description of the prohibitive construction, existential negation, and the use of the prefix $\{m a-\}$, a derivational suffix that has its origin in the proto-form * ma-, and the 'attributive' $\{k a-\}$. The analysis is based on primary data collected in some fieldwork with speakers of the Mehinaku language, from the Utawana and Kaupüna villages, in the Xingu National Park, Mato Grosso.

Keywords: Arawak languages; Mehinaku language; standard negation; typology of negation.

\section{Introdução ${ }^{3}$}

O Mehinaku, ISO 639-3: mmh, é uma língua indígena da família Arawak falada por, aproximadamente, 300 pessoas, distribuídas em quatro aldeias, são elas: Uyaipiyuku, Utawana, Aturua e Kaupüna, além do Posto Indígena de Vigilância (PIV)-Kurisevo. As quatro aldeias e o PIV se localizam na direção da fronteira sul do Território Indígena do Xingu, no estado do Mato Grosso.

Na classificação interna da família Arawák, o Mehinaku, assim como o Waurá e Yawalapiti, é inserido no grupo Pareci-Xingu, subgrupo Xinguano (AIKHENVALD, 1999) ou, no subgrupo Arawák Oriental (PAYNE, 1991; RAMIREZ, 2001). Tipologicamente, a língua Mehinaku espraia a ordem sintática $\mathrm{AV}(\mathrm{X}) \mathrm{O}$ nas sentenças transitivas declarativas simples, como se vê em (1):

3 A realização deste trabalho não seria possível sem a ajuda de Waxamani Mehinaku (aldeia Kaupüna) e de Yuta Mehinaku (aldeia Utawana). A eles, o nosso sincero agradecimento. 
(01)

$\begin{array}{llll}\text { a. küxü } & \text { ãitxa-pai } & \text { ata } & \text { ü-tai } \\ \text { tucano } & \text { 3.comer-IMPF } & \text { árvore } & \text { 3-fruta }\end{array}$

'o tucano está comendo frutinha' (Lit. 'o tucano come/está comendo a fruta da árvore')

$\begin{array}{llll}\text { b. txawaka } & \text { n-iya } & \text { kupatü-taku, } & \text { n-üpa } \\ \text { ontem } & \text { 1-ir.PSD } & \text { peixe-LOc } & \text { 1-ver.PSD } \\ \text { ü-nai } & \text { yanumaka } & \\ \text { 3-lugar } & \text { onça } & \\ \text { 'ontem fui na pesca, vi, nesse lugar, uma onça' }\end{array}$

Em construções com predicados ativos, a ordem é SUJEITO VERBO (cf. 02a); já com predicados estativos a ordem é VERBO SUJEITO (como em 02b, 02c):

(02)
a. ipiehü pulu-ka-wene-le un-iã
capivara pular-vBZ-rio-FUT água-LOC
'a capivara vai pular na água do rio'
b. ata ü-tawana-itsa-pai wakuwaku
árvore 3-ramo-LOC-IMPF tamanduá.bandeira
'o tamanduá bandeira está no ramo da árvore'
c. kau-pai nu-putü
dor-IMPF 1-coxa
'minha coxa está doendo'

\begin{abstract}
4 ABREVIATURAS: 1=primeira pessoa, 2=segunda pessoa, 3=terceira pessoa, ATB=atributivo, CAUS=causativo, $\quad \mathrm{COND}=$ condicional, $\mathrm{DAT}=$ dativo, $\mathrm{DCL}=$ declarativo, $\mathrm{DEM}=$ demonstrativo, $D I M=$ diminutivo, $E N F=e n f a ́ t i c o, E S T=e s t a t i v o, F E M=f e m i n i n o, \quad F R U S T=$ frustrativo, $F U T=$ futuro, IMPF=imperfeito, IMPSL=impessoal, LOC=locativo, NEG=negação, NUM=numeral, O=objeto, $P E R F=$ perfectivo, $P L=$ plural, POSS=posse, $P O T=$ potencial, PRIV=privativo, $P R O H=$ proibitivo, $\mathrm{PRON}=$ pronominal, $\mathrm{PSD}=$ passado, $\mathrm{RFL}=$ reflexivo, $\mathrm{SG}=$ singular, TOP=tópico, TRSF=transformativo, VBZ=verbalizador.
\end{abstract}


Com o intuito de contribuir para os estudos da língua Mehinaku, nesse texto, abordamos a negação, desde sua manifestação padrão, até outros tipos do mesmo fenômeno que a língua apresenta. Nesse sentido, além da introdução, este texto é composto de nove seções principais, as quais detalhamos, a seguir: na seção 2, considera-se brevemente o conceito de negação padrão e da forma como ela se manifesta na língua Mehinaku; na seção 3, apresenta-se a estrutura morfológica da negação em Mehinaku, relacionada aos tipos negação potencial, negação de evento inacabado e a negação asseverativa; na seção 4, abordam-se as características principais da negação proibitiva; a negação existencial é descrita na seção 5; a negação predicativa possessiva é tópico da seção 6; na seção 7, inclui-se a descrição da negação indefinida; na seção 8, analisa-se o morfema atributivo ka-e, sua contraparte, o privativo ma-, este é descrito na função de marcador de negação e, por fim, na seção 9, é descrito o paradigma simétrico na língua Mehinaku. As conclusões e referências encerram o texto.

No que diz respeito à metodologia, ressaltamos que os dados que apresentamos são primários, ou seja, foram coletados a partir da colaboração entre falantes Mehinaku e os autores deste trabalho e nossa análise é baseada nos pressupostos teóricos postulados sobre negação, na teoria linguística, por autores como Dahl (1979), Miestamo (2000, 2005, 2007), Dryer (1988), dentre outros.

\section{Negação padrão}

O termo negação padrão aparece, inicialmente, no artigo de Payne (1985) e é usado para se referir às formas básicas que uma determinada língua usa para negar uma sentença afirmativa ou, nas palavras do autor, "[...] entendemos que esse tipo de negação pode ser aplicado às sentenças mais mínimas e básicas. Tais sentenças são tipicamente orações principais e consistem em um único predicado com o menor número possível de substantivos e modificadores adverbiais" (PAYNE, 1985, p. 198, tradução nossa ${ }^{5}$ ). A partir de Miestamo (2005, 2007), o termo negação padrão passa a ser entendido como a negação da oração matriz em relação a um predicado verbal. Assume-se que esse tipo de construção é pragmaticamente neutro. Segundo Dahl (1979), há duas formas de expressar a negação: (i) de maneira morfológica e (ii) de maneira sintática. A negação morfológica se manifesta a partir da ocorrência de afixos flexionais, concretizados como prefixos, sufıxos, infixos, modificação da base, reduplicação, modificação prosódica, e, como característica mais comum, verifica-se, ainda, a ocorrência de um afıxo negativo preso ao verbo. Na negação sintática, o marcador que indica a negação é uma partícula ou um auxiliar, que acompanha outro verbo.

5 No original: "[...] we understand that type of negation that can apply to the most minimal and basic sentences. Such sentences are characteristically main clauses, and consist of a single predicate with as few noun phrases and adverbial modifiers as possible". 
Tanto os estudos sintáticos quanto os tipológicos têm focalizado a posição do marcador de negação na sentença, ou seja, as discussões se dão em torno da posição da negação em relação ao verbo. Assim, Dryer (1988) correlaciona a ordem dos constituintes e a posição da negação, considerando os três elementos principais da sentença sujeito (s), objeto (o) e verbo (v), tomando como amostra dados de 345 línguas. O estudo desse autor revela que as línguas podem registrar quatro subtipos de posicionamento da negação, como se mostra para as línguas svo e sov em (03):
a. Línguas svo: Negsvo, snegvo, svNegO, svoneg
b. Línguas Sov: Negsov, snegov, sonegv, sovNeg

O estudo de Dryer mostra, igualmente, que as línguas com a ordem de núcleo inicial diferem das línguas svo e sov, pois essas não atestam o total das possibilidades lógicas de colocação da negação. Ele mostra que todas as línguas vso e vos, consideradas em seu estudo, mostram apenas Negvso nas línguas vso e Negvos nas línguas de ordem vos.

Outro aspecto importante a se considerar nas construções relacionadas à negação padrão é se ela é simétrica ou, pelo contrário, assimétrica. Essa assimetria, proposta inicialmente por Miestamo (2000, 2005), refere-se à observação da coocorrência de outros marcadores de negação, além da construção negativa, ou seja, se a sentença se diferencia estruturalmente ou não de sua correspondente declarativa afirmativa. Uma construção negativa simétrica não difere de sua correspondente não negativa a não ser pela presença de marcadores que indicam a negação. Nela, os membros da construção afırmativa se manifestam numa correspondência biunívoca, ou seja, um-para-um. De maneira oposta, em uma construção negativa assimétrica ocorrem diferenças estruturais adicionais, que a distinguem de sua contraparte afirmativa. Nesse caso, os membros dos dois tipos de construção não mantêm uma correspondência biunívoca. As formas simétricas ou assimétricas podem ocorrer tanto nas construções sintáticas quanto nos paradigmas verbais.

O contraste entre esses dois tipos de construções negativas, a saber, simétrico e assimétrico, pode ser visto nos dados do espanhol (04) e do Awajún (Jívaro) em (05).
a. El perro está ladrando
b. El perro no está ladrando 
(05)

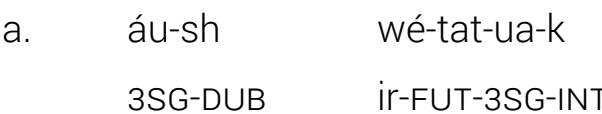
'ele também irá? (Interrogação dubitativa)
b. atsá áu-k wé-cha-ta-wa-i
NEG $\quad$ 3SG-TOP ir-NEG-FUT-3SG-DCL
'não, ele não irá'

\subsection{A negação padrão em Mehinaku}

A negação padrão na língua Mehinaku se dá pela ocorrência da partícula negativa aitsa. Esta partícula se manifesta em posição pré-verbal, como se observa nos seguintes dados:

$\begin{array}{lll}\text { n-ãitxa-pai } & \text { iyumu } & \text { ü-nühütü } \\ \text { 1-comer-IMPF } & \text { mutum } & \text { 3SG-carne.PosS }\end{array}$

'eu como carne de mutum'

$\begin{array}{llll}\text { aitsa } & \text { n-ãitxa-pai } & \text { yaapa } & \text { ü-nühütü } \\ \text { NEG } & \text { 1-comer-IMPF } & \text { paca } & \text { 3SG-carne.PosS }\end{array}$

'eu não como carne de paca'

$\begin{array}{llll}\text { txawaka } & \text { n-unupa } & \text { pawitsa } & \text { walamã } \\ \text { ontem } & \text { 1-ver.PSD } & \text { NUM.1 } & \text { sucuri }\end{array}$

'ontem vi uma sucuri'

aitsa n-unupa teme-pi-hã

NEG 1-ver.PSD jiboia-CL.cumprido-ENF

'não vi (uma) jiboia'

Em construções com verbos intransitivos ativos, a partícula aitsa 'NEG' também ocorre em posição pré-verbal, como mostramos no seguinte exemplo: 
(08)

$\begin{array}{llll}\text { nu-peku-yete } & \text { iya-la } & \text { nakai } & \text { ü-u-hã } \\ \text { 1-amigo-? } & \text { 3.ir-FUT } & \text { festa } & \text { 3-DAT-ENF }\end{array}$

'meu amigo irá à festa'

$\begin{array}{lllll}\text { nu-peku-yete } & \text { aitsa } & \text { iya-wa } & \text { nakai } & \text { ü-u-hã } \\ \text { 1-amigo-? } & \text { NEG } & \text { 3.ir-PERF } & \text { festa } & \text { 3-DAT-ENF }\end{array}$

'meu amigo não vai à festa'

Os dados em (09) exibem uma construção estativa. Note que a partícula aitsa 'NEG' posiciona-se antes da palavra que indica a propriedade que foi atribuída. Vejamos:

$\begin{array}{lll}\text { awitsiri-pai } & \text { n-itsu-pa-lu } & \text { ü-tulũ-hã } \\ \text { limpo-IMPF } & \text { 1-filha-EST-FEM } & \text { 3SG-orelha-ENF }\end{array}$

'a orelha de minha filha está limpa'

$\begin{array}{llll}\text { aitsa } & \text { awitsiri-pai } & \text { n-itsu-pa-lu } & \text { ü-tulũ-wi-ku } \\ \text { NEG } & \text { limpo-IMPF } & \text { 1-filha-EST-FEM } & \text { 3SG-orelha-PERF-DECL }\end{array}$

'a orelha de minha filha não está limpa'

Na língua Mehinaku, assim como no Wauja (BALL, 2014), ao ocorrerem duas orações, a partícula negativa aitsa 'NEG' precede o verbo da segunda construção, após o SN-sujeito dessa construção:

\begin{tabular}{|c|c|c|c|}
\hline tünexu-nãu & patã & a-tuluka-pai & Yamurikumã \\
\hline mulher-PL & somente & VBZ-dança-IMPF & Yamurikumã \\
\hline
\end{tabular}

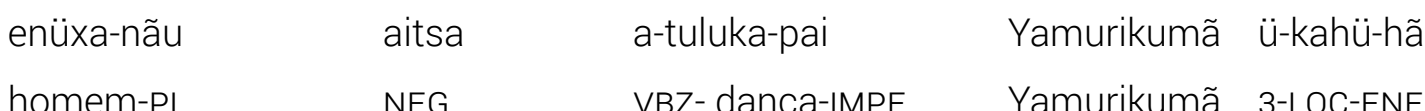
'somente as mulheres dançam na festa Yamurikumã, os homens não dançam na festa Yamurikumã' 


\section{Estrutura morfológica da negação em Mehinaku}

A partícula de negação aitsa modifica sua estrutura morfológica dependendo do contexto pragmático, o que diferencia a sua ocorrência em relação à negação padrão. Essa combinação de aitsa com determinados sufıxos ocorre quando o falante envolvido na comunicação dá resposta negativa a uma determinada questão levantada pelo locutor. Assim, por exemplo, em resposta a determinadas perguntas, a partícula aitsa se combina com o sufixo - wa, marcador de aspecto perfectivo, para compor uma resposta mais polida, delicada, cordial, como mostram os exemplos em (11):

a.

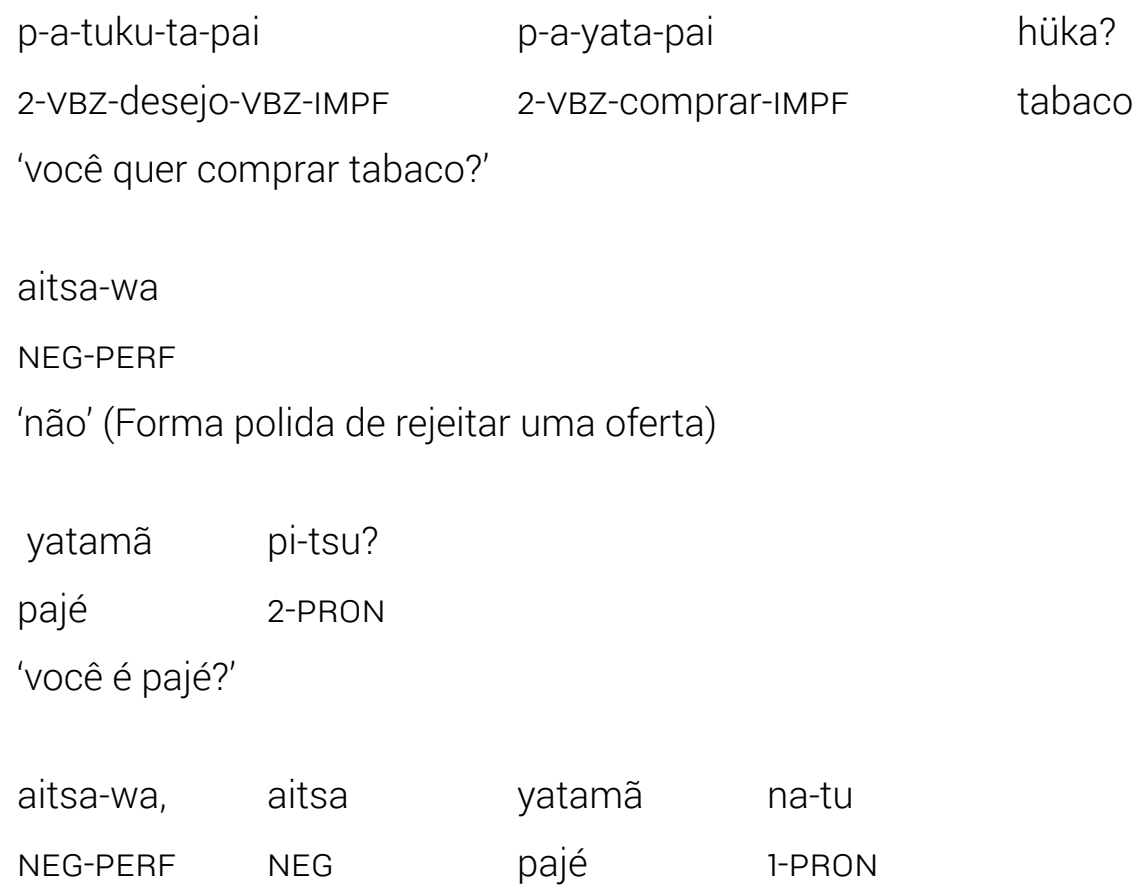

'não, não vai chover. Ontem já choveu' 

d. hiritxi-nãu patã a-yana-tuwa-pai ü-tenu-hã, velho-PL somente VBZ-pintura-RFL-IMPF 3-com-ENF
$\begin{array}{ll}\text { yamuku-tü-pa-nãu } & \text { aitsa-wa-hã } \\ \text { jovem-CL.cilíndrico-EST-PL } & \text { NEG-PERF-ENF }\end{array}$
'somente os velhos se pintam com ela (tipo de pintura), os jovens não'

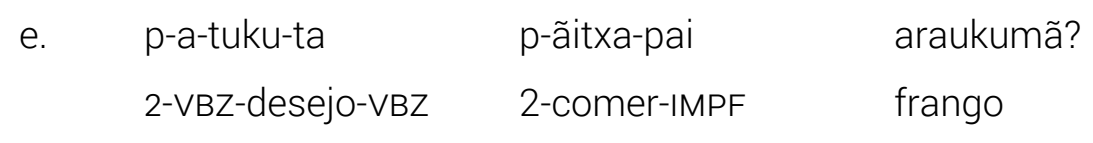

'você quer comer frango?'

\begin{tabular}{|c|c|c|c|}
\hline aitsa-wa, & aitsa & n-a-tuku-ta & n-ãitxa-pai \\
\hline NEG-PERF & NEG & 1-VBZ-desejo-VBZ & 1-comer-IMPF frango \\
\hline
\end{tabular}

'não, não quero comer frango'

Além da combinação de aitsa com o sufixo - wa, 'marcador de aspecto perfectivo', esta partícula pode combinar-se com outros sufixos, conforme trataremos a seguir.

\subsection{Negação potencial}

As construções potenciais ou de possibilidade são expressas pelo sufixo -miyã. Nos dados em (12), temos, então, um exemplo de evento condicional expresso pelo uso do referido sufixo:

$\begin{array}{lll}\text { p-iya-miyã } & \text { p-exuhü-ta-wa } & \text { p-unupa-miyã kupatü } \\ \text { 2-ir-COND } & \text { 2-anzol-vBZ-PERF } & \text { 2-ver-cond peixe }\end{array}$

muluku ü-tenu

coral 3-com

'se você vai pescar, talvez você veja o peixe com a cobra coral'

Nesse sentido, para se expressar negação com respeito a um evento condicional, em Mehinaku, combina-se a partícula de negação aitsa e o sufıxo -miyã. Os exemplos de (13) -(17) mostram os casos de construções de negação potencial: 


$\begin{array}{lllll}\text { aitsa-miyã } & \text { ãitxe-nü } & \text { aitsa-miyã } & \text { iya-kehe-nü } & \text { walame-pei } \\ \text { NEG-COND } & \text { comer-PERF } & \text { NEG-COND } & \text { 3.ir-convertir-PERF } & \text { sucuri-TRSF }\end{array}$

'se ele não comesse (o ovo da cobra sucuri), ele não ia se transformar em sucuri'

aitsa-miyã unü taka matamuyaka a-ya-la yakaku-yã-hã

NEG-COND água cair amanhã IPL-ir-FUT mato-LOC-ENF

'se não chover amanhã, nós iremos caçar'

$\begin{array}{llll}\text { aitsa-miyã } & \text { tuwa } & \text { p-a-tulu-ka-wi-ku } & \text { üneune-nẽu } \\ \text { NEG-COND } & \text { ir.RFL } & \text { 2-VBZ-dança-VBZ-PERF-DCL } & \text { pessoa-PL } \\ \text { ta-miyã } & \text { kira-pa } & \text { pi-txahü-ku-hã } & \\ \text { ir-COND } & \text { ficar-EST } & \text { 2-junto-DCL-ENF } & \end{array}$

'se você não vai dançar, as pessoas vão ficar reclamando de você'

(16)

$\begin{array}{lll}\text { xahã } & \text { aitsa-miyã } & \text { ünü-xü-wa } \\ \text { DEM } & \text { NEG-POT } & \text { 3-pai-PERF }\end{array}$

'ela não seria pai dele'

$\begin{array}{llll}\text { üxü-pai } & \text { aitsa-miyã } & \text { küna-wa } & \text { yakakũ-yã-hã } \\ \text { DEM-IMPF } & \text { NEG-POT } & \text { caçar-PERF } & \text { mato-Loc-ENF }\end{array}$

'hoje ninguém caçaria no mato'

\subsection{Negação de evento inacabado}

Outro tipo de construções negativas encontradas em Mehinaku são aquelas formadas pela partícula aitsa e pelo sufixo temporal -tüka 'ainda'. Essa forma de negação indica uma ação ainda não concluída ou ainda não iniciada (cf. 21). Os seguintes exemplos mostram esse tipo de construção: 
(18)
aitsa-tüka
n-ãitxa-pai,
n-ãitxa-tüka-i
neg-ainda
1-comer-impf
1-comer-ainda-impf

'ainda não comi, estou comendo ainda'
aitsa-tüka
üxü
ata-tai
ü-yuna-pai-hã
NEG-ainda
DEM
árvore-fruta
3-verde-IMPF-ENF

'esta fruta ainda não está madura'

aitsa-tüka kula-ta-ya-pai

NEG-ainda quente-VBZ-CL.líquido-IMPF

'(a água) ainda não esquentou'

$\begin{array}{lll}\text { aitsa-tüka } & \text { n-imi-tsa-pai } & \text { xepi-hã } \\ \text { NEG-ainda } & \text { 1-fazer-VBZ-IMPF } & \text { banco-ENF } \\ \text { 'ainda não fız o banco' } & \end{array}$

\subsection{Negação asseverativa}

Nesse tipo de negação, o falante tem certeza sobre a proposição, que é uma construção negativa. Assim, a negação é formada por aitsa e a ela é adjungido o sufixo indicativo de certeza -ya, para indicar uma avaliação negativa incisiva. Os exemplos seguintes ilustram esse tipo de negação:

$\begin{array}{llll}\text { yamuku-nãu, } & \text { aitsa } & \text { y-unupa } & \text { nu-peku-yete? } \\ \text { criança-PL } & \text { NEG } & \text { 2PL-VER.PSD } & \text { 1-amigo -? }\end{array}$

'crianças, vocês não viram meu amigo?'

$\begin{array}{llll}\text { aitsa-ya } & \text { a-unupa-wü } & \text { atsa } & \text { pu-peku-yete-pei? } \\ \text { NEG-certeza } & \text { IPL-ver.PSD-30 } & \text { quem } & \text { 2-amigo -? -IMPF }\end{array}$

'não, não o vimos. Quem é teu amigo?' 


$\begin{array}{llll}\text { nu-peku-yete } & \text { pu-muka-ta-i } & \text { nu-u } & \text { yana-tü? } \\ \text { 1-amigo-? } & \text { 2-arrumar-VBZ-IMPF } & \text { T-DAT } & \text { jenipapo-CL.cilíndrico }\end{array}$

'meu amigo, você arruma bambu para mim?

aitsa-ya nu yana-tü-ku

NEG-certeza 1 jenipapo-CL.cilíndrico-DCL

'não tenho bambu mais'

$\begin{array}{llll}\text { aitsa-ya } & \text { nu-tata-pai } & \text { n-üwa } & \text { putaka-naku-tsai } \\ \text { NEG-certeza } & \text { 1-saber-IMPF } & \text { 1-sobrinho } & \text { aldeia-CL.interior-Loc }\end{array}$

'não sei se meu sobrinho está na aldeia'

$\begin{array}{llll}\text { aitsa-ya } & \text { nu-tata-pai } & \text { üxe } & \text { yamuku-tü-pa-lu } \\ \text { NEG-certeza } & \text { 1-conhecer-IMPF } & \text { DEM } & \text { moça-CL.cilíndrico-EST-FEM }\end{array}$

'não conheço essa moça'

\section{Negação proibitiva}

As construções proibitivas se assemelham à negação padrão, pois também expressam negação por meio de uma partícula independente, neste caso, a partícula amiya. A negação proibitiva é o contrário do imperativo afirmativo. Nos exemplos subsequentes, temos construções negativas com a partícula amiya:

amiya

$$
\text { p-a-tuku-ta }
$$

рара

$\mathrm{PROH}$

2-VBZ-chamada-vBZ

pai

'não chame meu pai'

$$
\begin{array}{ll}
\text { amiya } & \text { p-uma-ta-wü } \\
\text { PROH } & \text { 2-fazer-caus-30 }
\end{array}
$$

'não faça isso' 
(28)

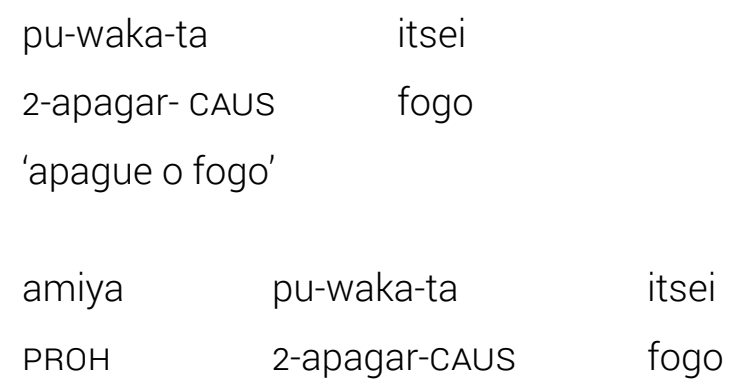

'não apague o fogo'

y-ãitxa üxe ulei-tsi-ku

2PL-comer DEM mandioca-CL.semente-DCL

'(vocês) comam essa mandioca'

amiya $\quad y$-ãitxa üxe ulei-tsi-ku

$\mathrm{PROH}$ 2PL-Comer DEM mandioca-CL.semente-DCL

'não comam essa mandioca'

\section{Negação existencial}

Nos dados de que dispomos, até então, não encontramos verbos existenciais na língua, entretanto, em construções existenciais negativas, eles são interpretados implicitamente pelo contexto. Dessa forma, as construções desse tipo são formadas pelo morfema aitsa 'NEG', em posição inicial, antes da proposição afirmativa, como se pode observar nos seguintes dados:

amuniya-pai autü-hã

muito-IMPF tronco-ENF

'há muitos troncos grossos'

aitsa amuniya autü-ku-hã

NEG muito tronCo-DCL-ENF

'não há muitos troncos grossos' 
(31)
amuniya-pai
tünexu-tãi-yãu
putaka-naku-wi-ku-hã
muitos-IMPF
mulher-DIM-PL
aldeia-LOC-PERF-DCL-ENF

'há muitas meninas na aldeia'

$\begin{array}{llll}\text { aitsa } & \text { amuniya-pai } & \text { enüxa-nãu } & \text { nakai ü-kahü-hã } \\ \text { NEG } & \text { muito-IMPF } & \text { homem-PL } & \text { festa } \\ \text { 3-LOC-ENF }\end{array}$

'não há muitos homens na festa'

$\begin{array}{lll}\text { amuniya-waxü-pai } & \text { kupatü } & \text { wene-naku-wi-ku } \\ \text { muito-de.verdade-IMPF } & \text { peixe } & \text { rio-CL.LOC-PERF-DCL }\end{array}$

'há de verdade muito peixe no rio'
aitsa
amuniya
kupatü-ku
wene-naku-wi-ku-hã
NEG
muito
peixe-DCL
riO-CL.LOC-PERF-DCL-ENF

'não há muito peixe no rio'

\section{Negação predicativa possessiva}

As construções de negação predicativa possessiva seguem o padrão da negação canônica, contudo, nesse caso, a partícula negativa aitsa se manifesta precedendo o possuidor. Esse tipo de construção é mostrado a seguir:

$\begin{array}{lll}\text { mipiyama-pai } & \text { papa } & \text { in-itsu-pa-lu-nãu } \\ \text { NUM.2-IMPF } & \text { 1.pai } & \text { 3-filha-EST-FEM-PL }\end{array}$

'meu pai tem duas filhas'

$\begin{array}{llll}\text { aitsa } & \text { mipiyama-pai } & \text { papa } & \text { in-itsu-pa-lu-nãu } \\ \text { NEG } & \text { NUM.2-IMPF } & \text { 1.pai } & \text { 3-filha-EST-FEM-PL }\end{array}$

'meu pai não tem duas filhas' 
$\begin{array}{ll}\text { ka-püxü-pai } & \text { nü-xe-xu } \\ \text { ATB-namorado-IMPF } & \text { 1-irmã-FEM }\end{array}$

'minha irmã (mais jovem) tem namorado'

aitsa ka-püxü-pai nü-xe-xu

NEG ATB-namorado-IMPF 1-irmã-FEM

'minha irmã (mais nova) não tem namorado'

\section{Negação indefinida}

Assim como vimos nas construções precedentes, a negação indefinida também se manifesta mediante o emprego da partícula de negação, a mesma que ocorre em posição pré-verbal. O sentido desse tipo de construção é dado pelo contexto, uma vez que na língua não há um elemento específico que indique o pronome indefinido. Vejamos os seguintes exemplos:

$\begin{array}{lll}\text { üxe-pai } & \text { aitsa } & \text { putuka-küna-wa-hã } \\ \text { DEM-IMPF } & \text { NEG } & \text { chegar.PSD-IMPSL-PERF-ENF }\end{array}$

'ninguém chegou hoje'

$\begin{array}{lll}\text { aitsa } & \text { aw-ãitxa-yete } & \text { ipiehü-ku-hã } \\ \text { NEG } & \text { IPL-comer-? } & \text { capivara-DCL-ENF }\end{array}$

'nós nunca comemos capivara'

$\begin{array}{llll}\text { aitsa } & \text { n-unupa-tãi } & \text { üneuneu } & \text { wene-naku-wi-ku } \\ \text { NEG } & \text { 1-ver-FRUST } & \text { gente } & \text { rio-LOC-PERF-DCL }\end{array}$

'não vi ninguém no rio'

$\begin{array}{ll}\text { aitsa } & \text { aw-ãitxa-lü } \\ \text { NEG } & \text { IPL-comer-FUT }\end{array}$

'não haverá nada para nós comermos' 
(40)

$\begin{array}{lll}\text { aitsa } & \text { n-upa-yete } & \text { teme } \\ \text { NEG } & \text { 1-ver.PSD-? } & \text { anta }\end{array}$

'nunca vi uma anta'

$\begin{array}{llll}\text { aitsa } & \text { yamuku-nãu } & \text { ãitxa } & \text { araukumã } \\ \text { NEG } & \text { criança-PL } & \text { comer.PSD } & \text { frango' }\end{array}$

$\begin{array}{llll}\text { aitsa-ya } & \text { n-unupa } & \text { üneuneu } & \text { waku } \\ \text { NEG-certeza } & \text { 1-ver.PSD } & \text { pessoa } & \text { rio.LOC }\end{array}$

'não vi ninguém no banho'

\section{Negação lexical}

Definimos como negação lexical as construções em que uma determinada base nominal contenha os prefixos derivacionais ka- 'atributivo' e/ou ma- 'privativo', fato que também é observado em outras línguas da mesma família. Os seguintes dados mostram a ocorrência dos prefixos citados:

nu-ka-nu-pai

1-ATR-mulher-IMPF

'tenho esposa'

$\begin{array}{ll}\text { aitsa } & \text { nu-ka-nu-pai } \\ \text { NEG } & \text { T-ATR-mulher-IMPF }\end{array}$

'não tenho esposa' 
(44)

nu-ke-me-pei

1- ATR -marido- IMPF

'tenho marido'

aitsa nu-ke-me-pei

NEG 1-ATR-marido-IMPF

'não tenho marido'

$\begin{array}{lll}\text { aitsa } & \text { k-uleke-pei } & \text { heritxa-ku-hã } \\ \text { NEG } & \text { ATR-comida-IMPF } & \text { velha-DCL-ENF }\end{array}$

'a velha não tem comida

(46)

$\begin{array}{lll}\text { aitsa } & \text { nu-peku-yete } & \text { ka-wana-pai-hã } \\ \text { NEG } & \text { 1-amigo-? } & \text { ATR-braço-IMPF-ENF }\end{array}$

'meu amigo não tem um braço'

(47)

$\begin{array}{llll}\text { xa } & \text { yamukuhĩ } & \text { aitsa } & \text { ka-nupüna-pai-hã } \\ \text { DEM } & \text { menino } & \text { NEG } & \text { ATR-nome-IMPF-ENF }\end{array}$

'aquele menino não tem nome'

(48)

José aitsa ka-tulũ-naku-pai ma- tulũ-naku-wa-hã

José neg atr-orelha-cl.loc-impf priv-orelha-loc-perf-enf

'José não ouve, ele é surdo'

(49)

$\begin{array}{ll}\text { ma-nu-wa-tüka } & \text { nü-tanule } \\ \text { PRIV-esposa-pessoa-ainda } & \text { 1-primo }\end{array}$

'meu primo ainda é solteiro' (Lit. meu primo ainda não tem esposa) 


\section{Paradigma simétrico}

Consoante com Miestamo (2000, 2005), encontramos nos paradigmas simétricos uma correspondência de um-para-um entre os membros que constituem os paradigmas afirmativos e negativos nas línguas. Nos exemplos a seguir (50-52), é possível observar que há simetria entre os paradigmas afırmativos e negativos nas construções com verbos transitivo e intransitivo (ativo e estativo) na língua Mehinaku:

\begin{tabular}{|c|c|c|c|}
\hline \multicolumn{2}{|c|}{ Afirmativo } & \multicolumn{2}{c|}{ Negativo } \\
\hline n-apai-tsa-pai & 'eu canto' & aitsa n-apai-tsa-pai & 'eu não canto' \\
\hline p-apai-tsa-pai & 'você canta' & p-apai-tsa-pai & 'você não canta' \\
\hline apai-tsa-pai & 'ele canta' & aitsa apai-tsa-pai & 'ele não canta' \\
\hline aw-apai-tsa-pai & 'nós cantamos' & aitsa aw-apai-tsa-pai & 'nós não cantamos' \\
\hline y-apai-tsa-pai & 'vocês cantam' & y-apai-tsa-pai & 'vocês não cantam' \\
\hline apai-tsa-pai-pia & 'eles cantam' & aitsa apai-tsa-pai-pia & 'eles não cantam' \\
\hline
\end{tabular}

(51)

\begin{tabular}{|c|c|c|c|}
\hline \multicolumn{2}{|c|}{ Afirmativo } & \multicolumn{2}{c|}{ Negativo } \\
\hline n-a-tuluka-pai & 'eu danço' & aitsa n-a-tuluka-pai & 'eu não danço' \\
\hline p-a-tuluka-pai & 'você dança' & aitsa p-a-tuluka-pai & 'você não dança' \\
\hline a-tuluka-pai & 'ele dança' & aitsa a-tuluka-pai & 'ele não dança' \\
\hline aw-a-tuluka-pai & 'nós dançamos' & aitsa aw-a-tuluka-pai & 'nós não dançamos' \\
\hline a-tuluka-pai-pia & 'eles dançam' & aitsa a-tuluka-pai-pia & 'eles não dançam' \\
\hline
\end{tabular}




\begin{tabular}{|c|c|c|c|}
\hline \multicolumn{2}{|c|}{ Afirmativo } & \multicolumn{2}{c|}{ Negativo } \\
\hline n-awitsiri-piai & 'eu sou bonita' & aitsa n-awitsiri-piai & 'eu não sou bonita' \\
\hline p-awitsiri-piai & 'você é bonita' & aitsa p-awitsiri-piai & 'você não é bonita' \\
\hline awitsiri-piai & 'ela é bonita' & aitsa awitsiri-piai & 'ela não é bonita' \\
\hline aw-awitsiri-piai & 'nós somos bonitas' & aitsa aw-awitsiri-piai & 'nós não somos bonitas' \\
\hline y-awitsiri-piai & 'vocês são bonitas' & aitsa y-awitsiri-piai & 'vocês não são bonitas' \\
\hline awitsiri-piai-pia & 'elas são bonitas' & aitsa awitsiri-piai-pia & 'elas não são bonitas' \\
\hline
\end{tabular}

\section{Conclusão}

Apresentamos uma descrição inicial das formas de ocorrência da negativa padrão e de outros tipos de negação na língua Mehinaku. Os dados analisados mostram que essa língua se caracteriza pelo uso da partícula de negação aitsa, marcador que ocupa a posição pré-verbal na construção sintática. Morfologicamente, a partícula aitsa pode se combinar com outros marcadores, os quais variam de acordo com as funções discursivo-pragmáticas na interação dos falantes Mehinaku. Esses marcadores que se combinam morfologicamente com a partícula de negação aitsa são sufixos relacionados às categorias de aspecto, modo e tempo. A ocorrência da partícula aitsa também se manifesta regularmente no paradigma verbal, tanto com verbos transitivos quanto com verbos intransitivos (ativo e estativo).

Como se pode deduzir, a partir dos dados analisados, a língua Mehinaku pode ser inserida tipologicamente no conjunto de línguas Arawák que apresentam o padrão simétrico de negação, tanto nas construções como nos paradigmas (MICHAEL; GRANADILLO, 2014). Além disso, observamos também que as construções proibitivas exibem uma organização semelhante à negação padrão e às construções imperativas afirmativas, porém, nesse tipo de construção, ocorre a partícula amiya ao invés de aitsa.

No artigo, outro aspecto abordado é o prefixo derivacional privativo ma- e o atributivo ka-. Ambos os morfemas derivacionais são recorrentes nas línguas da família Arawák (AIKHENVALD, 1999). 


\section{REFERÊNCIAS}

AIKHENVALD, A. Y. The Arawak language family. In: DIXON, R. M. W.; AIKHENVALD, A. Y. (ed.). The Amazonian languages. Cambridge: Cambridge University Press, 1999. p. 65-106.

BALL, C. Negation in Wauja discourse. In: MICHAEL, L.; GRANADILLO, T. (ed.). Negation in Arawak languages, 2014. p. 147-168.

DAHL, Ö. Typology of sentence negation. Linguistics, Berlin, v. 17, p. 79-106, 1979.

DERBYSHIRE, D. C.; PULLUM, G. K. (ed.). Handbook of Amazonian Languages. v. 3. Berlin: Mouton de Gruyter, 1991.

DIXON, R. M. W.; AIKHENVALD, A. Y. (ed.). The Amazonian languages. Cambridge: Cambridge University Press, 1999.

DRYER, M. S. Universals of negative position. In: HAMMOND, M.; MORAVCSIK, E. A.; WIRTH, J. (ed.). Studies in syntactic typology. Amsterdam/Philadelphia: John Benjamins, 1988. p. 93-124.

HAMMOND, M.; MORAVCSIK, E. A.; WIRTH, J. R. (ed.). Studies in syntactic typology (Typological studies in language, 17). Amsterdam/Philadelphia: John Benjamins, 1988.

MICHAEL, L.; GRANADILLO, T. (ed.). Negation in Arawak languages (Brill's Studies in the Indigenous Languages of the Americas, 6). Leiden, The Netherlands: Brill, 2014.

MIESTAMO, M. Towards a typology of standard negation. Nordic Journal of Linguistics, v. 23, p. 65-88, 2000.

MIESTAMO, M. Standard negation: the negation of declarative verbal main clauses in a typological perspective. Berlin: Mouton de Gruyter, 2005.

MIESTAMO, M. Negation - An overview of typological research. Language and Linguistic Compass, v. 1, n. 5, p. 552-570, 2007.

PAYNE, D. L. A classification of Maipuran (Arawakan) languages based on shared lexical retentions. In: DERBYSHIRE, D. C.; PULLUM, G. K. (ed.). Handbook of Amazonian Languages. v. 3. Berlin: Mouton de Gruyter, 1991. p. 355-499. 
PAYNE, J. R. Negation. In: SHOPEN, T. (ed.). Language typology and syntactic description, v. 1. Clause Structure. Cambridge: Cambridge University Press, 1985. p. 197-242.

RAMIREZ, H. Línguas Arawak da Amazônia Setentrional. Comparação e descrição. Manaus: EDUA, 2001.

SHOPEN, T. (ed.). Language typology and syntactic description, v. 1, Clause structure. Cambridge: Cambridge University Press, 1985. 